\title{
WEAK LINKING THEOREMS AND SCHRÖDINGER EQUATIONS WITH CRITICAL SOBOLEV EXPONENT
}

\author{
Martin Schechter $^{1, *}$ AND Wenming Zou ${ }^{2, \dagger}$
}

\begin{abstract}
In this paper we establish a variant and generalized weak linking theorem, which contains more delicate result and insures the existence of bounded Palais-Smale sequences of a strongly indefinite functional. The abstract result will be used to study the semilinear Schrödinger equation $-\Delta u+$ $V(x) u=K(x)|u|^{2^{*}-2} u+g(x, u), u \in W^{1,2}\left(\mathbf{R}^{N}\right)$, where $N \geq 4 ; V, K, g$ are periodic in $x_{j}$ for $1 \leq j \leq N$ and 0 is in a gap of the spectrum of $-\Delta+V ; K>0$. If $0<g(x, u) u \leq c|u|^{2^{*}}$ for an appropriate constant $c$, we show that this equation has a nontrivial solution.
\end{abstract}

Mathematics Subject Classification. 35B33, 35J65, 35Q55.

Received October 28, 2002.

\section{INTRODUCTION}

In this article, the aim is to study the following semilinear Schrödinger equation with critical Sobolev exponent and periodic potential:

$$
-\Delta u+V(x) u=K(x)|u|^{2^{*}-2} u+g(x, u), \quad u \in W^{1,2}\left(\mathbf{R}^{N}\right),
$$

where $N \geq 4 ; 2^{*}:=2 N /(N-2)$ is the critical Sobolev exponent and $g$ is of subcritical growth.

First of all, we recall that the equation

$$
-\Delta u+\lambda u=|u|^{2^{*}-2} u, \quad \lambda \neq 0,
$$

has only the trivial solution $u=0$ in $W^{1,2}\left(\mathbf{R}^{N}\right)(c f .[4])$. Therefore, the existence of nontrivial solution of $(\mathrm{S})$ is an interesting problem.

Before we state the main result, we introduce the following conditions:

$\left(\mathbf{S}_{1}\right) V, K \in \mathcal{C}\left(\mathbf{R}^{N}, \mathbf{R}\right), g \in \mathcal{C}\left(\mathbf{R}^{N} \times \mathbf{R}, \mathbf{R}\right), k_{0}:=\inf _{x \in \mathbf{R}^{N}} K(x)>0 ; V, K, g$ are 1-periodic in $x_{j}$ for $j=$ $1, \ldots, N$

$\left(\mathbf{S}_{2}\right) 0 \notin \sigma(-\Delta+V)$ and $\sigma(-\Delta+V) \cap(-\infty, 0) \neq \emptyset$, where $\sigma$ denotes the spectrum in $L^{2}\left(\mathbf{R}^{N}\right)$;

$\left(\mathbf{S}_{3}\right) K\left(x_{0}\right):=\max _{x \in \mathbf{R}^{N}} K(x)$ and $K(x)-K\left(x_{0}\right)=o\left(\left|x-x_{0}\right|^{2}\right)$ as $x \rightarrow x_{0}$ and $V\left(x_{0}\right)<0$;

\footnotetext{
Keywords and phrases. Linking, Schrödinger equations, critical Sobolev exponent.

${ }^{1}$ Department of Mathematics, University of California, Irvine, CA 92697-3875, USA; e-mail: mschecht@math.uci.edu

* Supported in part by a NSF grant.

2 Department of Mathematical Sciences, Tsinghua University, Beijing 100084, China; e-mail: wzou@math.tsinghua.edu.cn

† Supported by NSFC grant (GP10001019). This work was finished when W. Zou was visiting the University of California at Irvine during 2001-2004. He thanks all the members of the Math Department of UCI for offering this position. 
$\left(\mathbf{S}_{4}\right)|g(x, u)| \leq c_{0}\left(1+|u|^{p-1}\right)$ for all $(x, u) \in \mathbf{R}^{N} \times \mathbf{R}$, where $c_{0}>0$ and $p \in\left(2,2^{*}\right)$. Moreover, $g(x, u) /|u|^{2^{*}-1}$ $\rightarrow 0$ as $u \rightarrow 0$ uniformly for $x \in \mathbf{R}^{N}$;

$\left(\mathbf{S}_{5}\right) g(x, u) u>0$ for all $x \in \mathbf{R}^{N}$ and $u \neq 0$.

The main result is the following:

Theorem 1.1. Assume that $\left(\mathrm{S}_{1}-\mathrm{S}_{5}\right)$ hold. If

$$
\frac{k_{0}}{m_{g}} \geq \frac{N-2}{2}, \quad \text { where } \quad m_{g}:=\max _{x \in \mathbf{R}^{N}, u \in \mathbf{R} \backslash\{0\}} \frac{g(x, u) u}{|u|^{2 *}},
$$

then equation (S) has a solution $u \neq 0$. Particularly, if $K(x) \equiv k_{0}>0,\left(\mathrm{~S}_{3}\right)$ can be deleted and the same result holds.

An equivalent form of Theorem 1.1 is the following:

Corrolary 1.1. Assume that $\left(\mathrm{S}_{1}-\mathrm{S}_{5}\right)$ hold. Then the following Schrödinger equation

$$
-\Delta u+V(x) u=K(x)|u|^{2^{*}-2} u+\beta g(x, u), \quad u \in W^{1,2}\left(\mathbf{R}^{N}\right),
$$

has a nontrivial solution for all $\beta \in\left(0,2 k_{0} /\left(m_{g}(N-2)\right)\right]$. If $K(x) \equiv k_{0}>0$, condition $\left(\mathrm{S}_{3}\right)$ can be omitted and the same result holds.

Remark 1.1. It is an open problem whether or not the results of the present paper remain true for the case of $N=3$. This problem is also raised by Y.Y. Li in private communications.

Now we make some comments on this problem and the main results. Under the hypotheses on $V$ the spectrum of $-\Delta+V$ in $L^{2}\left(\mathbf{R}^{N}\right)$ is purely continuous and bounded below and is the union of disjoint closed intervals $(c f$. Th. XIII. 100 of [17] and Th. 4.5.9 of [13]), which makes the problem difficult to be dealt with.

Recently, equation (S) was studied in [6], which also generalized the early results obtained in [7]. In [6], the assumption

$$
0 \leq \gamma G(x, u) \leq u g(x, u) \text { on } \mathbf{R}^{N} \times \mathbf{R},
$$

where $\gamma=2 ; G(x, u):=\int_{0}^{u} g(x, s) d s$, was imposed in order to prove the boundedness of the Palais-Smale sequence. Obviously, this condition contains the case of $g \equiv 0$. Condition (1.3) has three disadvantages: the first is that one has to compute the primitive function $G$ of $g$; the second is that one has to check the second inequality of (1.3); the third is that (1.3) does not contain the sublinear (at infinity) case and some asymptotically linear (at infinity) case. But sometimes, it is either impossible to compute $G$ so that (1.3) can be checked or the second inequality of (1.3) does not hold.

These cases happen on the following three examples:

$$
\begin{aligned}
\text { (i) } g(x, u): & = \begin{cases}c|u|^{2^{*}} u \mathrm{e}^{-\sin ^{2} u} & |u| \leq 1 \\
c|u|^{-2 / 3} u \mathrm{e}^{-\sin ^{2} u}(1+\ln |u|) & |u| \geq 1,\end{cases} \\
\text { (ii) } g(x, u): & =\left\{\begin{array}{ll}
c|u|^{2^{*}} u & |u| \leq 1 \\
c|u|^{-2 / 3} u & |u| \geq 1,
\end{array}\right. \text { (sublinear at infinity) } \\
\text { (iii) } g(x, u): & = \begin{cases}c|u|^{2^{*}} u & |u| \leq 1 \\
\frac{c}{2}\left(u+|u|^{-2 / 3} u\right) & |u| \geq 1,\end{cases}
\end{aligned}
$$

However, we emphasize that the above examples satisfy the hypotheses of Theorem 1.1 of the present paper for appropriate $c>0$. Moreover, conditions $\left(\mathrm{S}_{4}\right)$ and $\left(\mathrm{S}_{5}\right)$ permit the nonlinearity $g$ to be superlinear, asymptotically linear or sublinear.

Evidently, if we set

$$
\bar{m}_{g}(r)=\max _{x \in \mathbf{R}^{N},|u| \geq r \text { or }|u| \leq 1 / r} \frac{g(x, u) u}{|u|^{2^{*}}},
$$


then $k_{0} / \bar{m}_{g}(r) \rightarrow \infty$ as $r \rightarrow \infty$. It is an open problem whether or not assumption (1.2) can be concealed or equivalently, Corollary 1.1 holds for all $\beta>0$. On the other hand, it should be mentioned that (1.2) is the price to pay for relaxing (1.3).

Equation (S) with $K(x) \equiv 0$, i.e., the nonlinear term is of subcritical growth, has been studied by several authors (for example, cf. $[1-3,5,8,10-12,24,26]$ and the references cited therein). In those papers, the Ambrosetti-Rabinowitz condition (1.3) with $\gamma>2$ was needed. In [23], the authors considered the asymptotically linear case. In [25] (see also [3]), zero is an end point of $\sigma(-\Delta+V)$. In [14], the author studied a special case $-\Delta u=K u^{5}$ in $\mathbf{R}^{3}$ (see also [15] for higher dimension case on $S^{N}$ ). Very little is known for (S) with critical Sobolev exponent and periodic potential.

Without (1.3) with $\gamma \geq 2$, the problem becomes more complicated. The main obstacle is how to get a bounded Palais-Smale sequence. To get over this road block, we establish a variant and generalized weak linking theorem. Roughly speaking, let $E$ be a Hilbert space, let $N \subset E$ be a separable subspace, and let $Q \subset N$ be a bounded open convex set, with $p_{0} \in Q$. Let $F$ be a "weak" continuous map of $E$ onto $N$ such that $\left.F\right|_{Q}=i d$ and that $F(u-v)-(F(u)-F(v))$ is contained in a fixed finite-dimensional subspace of $E$ for all $u, v \in E$. Then under suitable hypotheses, $\partial Q \operatorname{links} F^{-1}\left(p_{0}\right)$ with respect to a suitable restricted class of deformations of $\bar{Q}$. We will define a family of $\mathcal{C}^{1}$-functional $\left\{H_{\lambda}\right\}_{\lambda \in[1,2]}$ which is related to problem (S). Since the spectrum of $-\Delta+V$ in $L^{2}\left(\mathbf{R}^{N}\right)$ is purely continuous, both positive and negative subspaces of the functional $H_{\lambda}$ are infinite-dimensional. Moreover, $H_{\lambda}$ is unbounded from both below and above, the so-called strongly indefinite functional. Furthermore, because of the weaker assumptions (particularly, without (1.3)), the usual minimax techniques (e.g. $[1-7,10,11,14,15])$, can not be used here. However, by using the new weak linking theorem, we show that $\left\{H_{\lambda}\right\}$ has a bounded Palais-Smale sequence for almost every $\lambda \in[1,2]$. The main idea is the Monotonicity Trick due to $[10,11]$ (see also [21] for an earlier application). Other applications of this trick can be found in $[23,27,28,30-32]$. We also give the estimates of the energy, i.e., the energy lies in $\left[\inf _{F^{-1}\left(p_{0}\right)} H_{\lambda}, \sup _{\bar{Q}} H_{1}\right]$. By this way, there is no need to impose some strong conditions for proving the boundedness of Palais-Smale sequences. In other words, we permit much more freedom for the nonlinearity.

The paper is organized as follows: in Section 2, we establish a variant weak linking theorem. In Section 3, equation (S) will be studied. In Section 4, an Appendix will be given.

\section{A VARIANT WEAK LINKING THEOREM}

Let $E$ be a Hilbert space with norm $\|\cdot\|$ and inner product $\langle\cdot, \cdot\rangle$ and have an orthogonal decomposition $E=N \oplus N^{\perp}$, where $N \subset E$ is a closed and separable subspace. Since $N$ is separable, we can define a new norm $|v|_{w}$ satisfying $|v|_{w} \leq\|v\|, \forall v \in N$ and such that the topology induced by this norm is equivalent to the weak topology of $N$ on bounded subset of $N$ (see Appendix of Sect. 4). For $u=v+w \in E=N \oplus N^{\perp}$ with $v \in N, w \in N^{\perp}$, we define $|u|_{w}^{2}=|v|_{w}^{2}+\|w\|^{2}$, then $|u|_{w} \leq\|u\|, \forall u \in E$.

Particularly, if $\left(u_{n}=v_{n}+w_{n}\right)$ is $|\cdot|_{w}$-bounded and $u_{n} \stackrel{|\cdot|_{w}}{\rightarrow} u$, then $v_{n} \rightarrow v$ weakly in $N, w_{n} \rightarrow w$ strongly in $N^{\perp}, u_{n} \rightarrow v+w$ weakly in $E(c f .[9])$.

Let $Q \subset N$ be a $\|\cdot\|$-bounded open convex subset, $p_{0} \in Q$ be a fixed point. Let $F$ be a $|\cdot|{ }_{w}$-continuous map from $E$ onto $N$ satisfying

- $\left.F\right|_{Q}=i d ; F$ maps bounded sets to bounded sets;

- there exists a fixed finite-dimensional subspace $E_{0}$ of $E$ such that $F(u-v)-(F(u)-F(v)) \subset E_{0}, \forall v, u \in E ;$

- $F$ maps finite-dimensional subspaces of $E$ to finite-dimensional subspaces of $E$.

We use the letter $c$ to denote various positive constants.

$$
A:=\partial Q, \quad B:=F^{-1}\left(p_{0}\right),
$$

where $\partial Q$ denotes the $\|\cdot\|$-boundary of $Q$. 
Remark 2.1. There are many examples:

(i) let $N=E^{-}, N^{\perp}=E^{+}$, then $E=E^{-} \oplus E^{+}$and let $Q:=\left\{u \in E^{-}:\|u\|<R\right\}, p_{0}=0 \in Q$. For any $u=u^{-} \oplus u^{+} \in E$, define $F: E \mapsto N$ by $F u:=u^{-}$, then $A:=\partial Q, B:=F^{-1}\left(p_{0}\right)=E^{+}$satisfy the above conditions;

(ii) let $E=E^{-} \oplus E^{+}, z_{0} \in E^{+}$with $\left\|z_{0}\right\|=1$. For any $u \in E$, we write $u=u^{-} \oplus s z_{0} \oplus w^{+}$with $u^{-} \in E^{-}, s \in \mathbf{R}, w^{+} \in\left(E^{-} \oplus \mathbf{R} z_{0}\right)^{\perp}:=E_{1}^{+}$. Let $N:=E^{-} \oplus \mathbf{R} z_{0}$. For $R>0$, let $Q:=\{u:=$ $\left.u^{-}+s z_{0}: s \in \mathbf{R}^{+}, u^{-} \in E^{-},\|u\|<R\right\}, p_{0}=s_{0} z_{0} \in Q, s_{0}>0$. Let $F: E \mapsto N$ be defined by $F u:=u^{-}+\left\|s z_{0}+w^{+}\right\| z_{0}$, then $F, Q, p_{0}$ satisfy the above conditions with

$$
B=F^{-1}\left(s_{0} z_{0}\right)=\left\{u:=s z_{0}+w^{+}: s \geq 0, w^{+} \in E_{1}^{+},\left\|s z_{0}+w^{+}\right\|=s_{0}\right\}
$$

In fact, according to the definition, $\left.F\right|_{Q}=i d$ and $F$ maps bounded sets to bounded sets. On the other hand, for any $u, v \in E$, we write $u=u^{-}+s z_{0}+w^{+}, v=v^{-}+t z_{0}+w_{1}^{+}$, then

therefore,

$$
\begin{gathered}
F(u)=u^{-}+\left\|s z_{0}+w^{+}\right\| z_{0}, \quad F(v)=v^{-}+\left\|t z_{0}+w_{1}^{+}\right\| z_{0}, \\
F(u-v)=u^{-}-v^{-}+\left\|(s-t) z_{0}+w^{+}-w_{1}^{+}\right\| z_{0},
\end{gathered}
$$

$$
\begin{aligned}
F(u-v)-(F(u)-F(v)) & =\left(\left\|(s-t) z_{0}+w^{+}-w_{1}^{+}\right\|-\left\|s z_{0}+w^{+}\right\|+\left\|t z_{0}+w_{1}^{+}\right\|\right) z_{0} \\
& \subset \mathbf{R} z_{0}:=E_{0} \quad(\text { an } 1 \text {-dimensional subspace }) .
\end{aligned}
$$

For $H \in \mathcal{C}^{1}(E, \mathbf{R})$, we define

$\Gamma:=\left\{h:[0,1] \times \bar{Q} \mapsto E, h\right.$ is $|\cdot|_{w}$-continuous. For any $\left(s_{0}, u_{0}\right) \in[0,1] \times \bar{Q}$,

$$
\begin{aligned}
& \text { there is a }|\cdot|_{w}-\text { neighborhood } U_{\left(s_{0}, u_{0}\right)} \text { such that } \\
& \left\{u-h(t, u):(t, u) \in U_{\left(s_{0}, u_{0}\right)} \cap([0,1] \times \bar{Q})\right\} \subset E_{\mathrm{fin}}, \\
& \qquad h(0, u)=u, H(h(s, u)) \leq H(u), \forall u \in \bar{Q}\},
\end{aligned}
$$

then $\Gamma \neq \emptyset$ since $i d \in \Gamma$. Here and then, we use $E_{\text {fin }}$ to denote various finite-dimensional subspaces of $E$ whose exact dimensions are irrelevant and depend on $\left(s_{0}, u_{0}\right)$.

The variant weak linking theorem is:

Theorem 2.1. The family of $\mathcal{C}^{1}$-functional $\left(H_{\lambda}\right)$ has the form

$$
H_{\lambda}(u):=I(u)-\lambda J(u), \quad \forall \lambda \in[1,2] .
$$

Assume

(a) $J(u) \geq 0, \forall u \in E ; H_{1}:=H$;

(b) $I(u) \rightarrow \infty$ or $J(u) \rightarrow \infty$ as $\|u\| \rightarrow \infty$;

(c) $H_{\lambda}$ is $|\cdot|_{w}$-upper semicontinuous; $H_{\lambda}^{\prime}$ is weakly sequentially continuous on E. Moreover, $H_{\lambda}$ maps bounded sets to bounded sets;

(d) $\sup _{A} H_{\lambda}<\inf _{B} H_{\lambda}, \forall \lambda \in[1,2]$.

Then for almost all $\lambda \in[1,2]$, there exists a sequence $\left(u_{n}\right)$ such that

$$
\sup _{n}\left\|u_{n}\right\|<\infty, \quad H_{\lambda}^{\prime}\left(u_{n}\right) \rightarrow 0, \quad H_{\lambda}\left(u_{n}\right) \rightarrow C_{\lambda}
$$


where

$$
C_{\lambda}:=\inf _{h \in \Gamma} \sup _{u \in \bar{Q}} H_{\lambda}(h(1, u)) \in\left[\inf _{B} H_{\lambda}, \sup _{\bar{Q}} H\right]
$$

Before proving this theorem, let us make some remarks.

Remark 2.2. Similar weak linking was developed in [18-20,29]. In [18-20], conditions "F|$\left.\right|_{N} \equiv i d "$ and " $F(v-w)=v-F w$ for all $v \in N, w \in E$ " were stated but not needed. All that was used was $\left.F\right|_{Q} \equiv i d$ and $F(v-w)=v-F w$ for all $v \in Q, w \in E$. This was noted in [29]. Particularly, we emphasize that because the monotonicity trick was not used in [18-20,29], the boundedness of Palais-Smale sequence was not a consequence of the Theorems. Therefore, some compactness conditions were introduced and played an important role. The results of [18-20,29] can not be used to deal with equation (S).

Remark 2.3. In [12] (see also [26]), some theorems were given which contained only a particular linking and the boundedness of Palais-Smale sequence is also remained unknown. Therefore, in applications, AmbrosettiRabinowitz type condition (1.3) with $\gamma>2$ is needed. In [12,26], a $\tau$-topology is specially constructed to accommodate the splitting of $E$ into subspace and by this, a new degree of Leray-Schauder type is established. The new degree is also applied in $[23,25,27,28]$.

Proof of Theorem 2.1.

Step 1. We prove that $C_{\lambda} \in\left[\inf _{B} H_{\lambda}, \sup _{Q} H\right]$. Evidently, by the definition of $C_{\lambda}$,

$$
C_{\lambda} \leq \sup _{u \in \bar{Q}} H_{\lambda}(u) \leq \sup _{u \in \bar{Q}} H_{1}(u) \equiv \sup _{u \in \bar{Q}} H(u)<\infty
$$

To show $C_{\lambda} \geq \inf _{B} H_{\lambda}$ for all $\lambda \in[1,2]$, we have to prove that $h(1, \bar{Q}) \cap B \neq \emptyset$ for all $h \in \Gamma$. By hypothesis, the $\operatorname{map} F h:[0,1] \times \bar{Q} \rightarrow N$ is $|\cdot|_{w}$-continuous. Let $K:=[0,1] \times \bar{Q}$. Then $K$ is $|\cdot|_{w}$-compact. In fact, since $K$ is bounded with respect to both norms $|\cdot|_{w}$ and $\|\cdot\|$, for any $\left(t_{n}, v_{n}\right) \in K$, we may assume that $v_{n} \rightarrow v_{0}$ weakly in $E$ and that $t_{n} \rightarrow t_{0} \in[0,1]$. Then $v_{0} \in \bar{Q}$ since $\bar{Q}$ is convex. Since on the bounded set $Q \subset N$, the $|\cdot|_{w}$-topology is equivalent to the weak topology, then $u_{n} \stackrel{|\cdot|_{w}}{\rightarrow} v_{0}$. So, $K$ is $|\cdot|_{w}$-compact. By the definition of $\Gamma$, for any $\left(s_{0}, u_{0}\right) \in K$, there is a $|\cdot|_{w}$-neighborhood $U_{\left(s_{0}, u_{0}\right)}$ such that

$$
\left\{u-h(t, u):(t, u) \in U_{\left(s_{0}, u_{0}\right)} \cap K\right\} \subset E_{\text {fin }},
$$

here and then, we use $E_{\text {fin }}$ to denote various finite-dimensional subspaces of $E$ whose exact dimensions are irrelevant. Now, $K \subset \cup_{(s, u) \in K} U_{(s, u)}$. Since $K$ is $|\cdot|_{w}$-compact, $K \subset \cup_{i=1}^{j_{0}} U_{\left(s_{i}, u_{i}\right)},\left(s_{i}, u_{i}\right) \in K$. Consequently,

$$
\{u-h(t, u):(t, u) \in K\} \subset E_{\text {fin }} .
$$

Hence, by the basic assumptions on $F$,

$$
F\{u-h(t, u):(t, u) \in K\} \subset E_{\text {fin }}
$$

and

$$
\{u-F h(t, u):(t, u) \in K\} \subset E_{\text {fin }} .
$$

Then we can choose a finite-dimensional subspace $E_{\text {fin }}$ such that $p_{0} \in E_{\text {fin }}$ and that

$$
F h:[0,1] \times\left(\bar{Q} \cap E_{\text {fin }}\right) \rightarrow E_{\text {fin }} .
$$

We claim that $F h(t, u) \neq p_{0}$ for all $u \in \partial\left(\bar{Q} \cap E_{\text {fin }}\right)=\partial \bar{Q} \cap E_{\text {fin }}$ and $t \in[0,1]$. By way of negation, if there exist $t_{0} \in[0,1]$ and $u_{0} \in \partial \bar{Q} \cap E_{\text {fin }}$ such that $F h\left(t_{0}, u_{0}\right)=p_{0}$, i.e., $h\left(t_{0}, u_{0}\right) \in B$. It follows that

$$
H_{1}\left(u_{0}\right) \geq H_{1}\left(h\left(t_{0}, u_{0}\right)\right) \geq \inf _{B} H_{1}>\sup _{\partial \bar{Q}} H_{1},
$$


which contradicts the assumption (d). Thus, our claim is true. By the homotopy invariance of Brouwer degree, we get that

$$
\begin{aligned}
\operatorname{deg}\left(F h(1, \cdot), Q \cap E_{\text {fin }}, p_{0}\right) & =\operatorname{deg}\left(F h(0, \cdot), Q \cap E_{\text {fin }}, p_{0}\right) \\
& =\operatorname{deg}\left(i d, Q \cap E_{\text {fin }}, p_{0}\right) \\
& =1 .
\end{aligned}
$$

Therefore, there exists $u_{0} \in Q \cap E_{\text {fin }}$ such that $F h\left(1, u_{0}\right)=p_{0}$.

Step 2. Evidently, $\lambda \mapsto C_{\lambda}$ is nonincreasing, hence $C_{\lambda}^{\prime}=\frac{d C_{\lambda}}{d \lambda}$ exists for almost every $\lambda \in[1,2]$. We consider those $\lambda \in[1,2]$ where $C_{\lambda}^{\prime}$ exists and use the monotonicity trick (see e.g. [21]).

Let $\lambda_{n} \in[1,2]$ be a strictly increasing sequence such that $\lambda_{n} \rightarrow \lambda$. Then there exists $n(\lambda)$ large enough such that

$$
-C_{\lambda}^{\prime}-1 \leq \frac{C_{\lambda_{n}}-C_{\lambda}}{\lambda-\lambda_{n}} \leq-C_{\lambda}^{\prime}+1 \text { for } n \geq n(\lambda) .
$$

Step 3. There exists a sequence $h_{n} \in \Gamma, k:=k(\lambda)>0$ such that $\left\|h_{n}(1, u)\right\| \leq k$ if $H_{\lambda}\left(h_{n}(1, u)\right) \geq C_{\lambda}-\left(\lambda-\lambda_{n}\right)$. In fact, by the definition of $C_{\lambda_{n}}$, let $h_{n} \in \Gamma$ be such that

$$
\sup _{u \in \bar{Q}} H_{\lambda_{n}}\left(h_{n}(1, u)\right) \leq C_{\lambda_{n}}+\left(\lambda-\lambda_{n}\right) .
$$

Therefore, if $H_{\lambda}\left(h_{n}(1, u)\right) \geq C_{\lambda}-\left(\lambda-\lambda_{n}\right)$ for some $u \in \bar{Q}$, then for $n \geq n(\lambda)$ (large enough), by (2.1) and (2.2),

$$
\begin{aligned}
J\left(h_{n}(1, u)\right) & =\frac{H_{\lambda_{n}}\left(h_{n}(1, u)\right)-H_{\lambda}\left(h_{n}(1, u)\right)}{\lambda-\lambda_{n}} \\
& \leq \frac{C_{\lambda_{n}}-C_{\lambda}}{\lambda-\lambda_{n}}+2 \\
& \leq-C_{\lambda}^{\prime}+3
\end{aligned}
$$

and

$$
\begin{aligned}
I\left(h_{n}(1, u)\right) & =H_{\lambda_{n}}\left(h_{n}(1, u)\right)+\lambda_{n} J\left(h_{n}(1, u)\right) \\
& \leq C_{\lambda_{n}}+\left(\lambda-\lambda_{n}\right)+\lambda_{n}\left(-C_{\lambda}^{\prime}+3\right) \\
& \leq C_{\lambda}-\lambda C_{\lambda}^{\prime}+3 \lambda .
\end{aligned}
$$

By assumption (b), $\left\|h_{n}(1, u)\right\| \leq k:=k(\lambda)$.

Step 4. By step 2 and (2.2)

$$
\sup _{u \in \bar{Q}} H_{\lambda}\left(h_{n}(1, u)\right) \leq \sup _{u \in \bar{Q}} H_{\lambda_{n}}\left(h_{n}(1, u)\right) \leq C_{\lambda}+\left(2-C_{\lambda}^{\prime}\right)\left(\lambda-\lambda_{n}\right) .
$$

Step 5. For $\varepsilon>0$, define

$$
F_{\varepsilon}(\lambda):=\left\{u \in E:\|u\| \leq k+4,\left|H_{\lambda}(u)-C_{\lambda}\right| \leq \varepsilon\right\} .
$$

Then we claim, for $\varepsilon$ small enough, that $\inf \left\{\left\|H_{\lambda}^{\prime}(u)\right\|: u \in F_{\varepsilon}(\lambda)\right\}=0$. Otherwise, there exists $\varepsilon_{0}>0$ such that $\left\|H_{\lambda}^{\prime}(u)\right\| \geq \varepsilon_{0}$ for all $u \in F_{\varepsilon_{0}}(\lambda)$. Let $h_{n} \in \Gamma$ be as in Steps 3,4 and $n$ be large enough such that $\lambda-\lambda_{n} \leq \varepsilon_{0}$ and $\left(2-C_{\lambda}^{\prime}\right)\left(\lambda-\lambda_{n}\right) \leq \varepsilon_{0}$. Define

$$
F_{\varepsilon_{0}}^{*}(\lambda):=\left\{u \in E:\|u\| \leq k+4, C_{\lambda}-\left(\lambda-\lambda_{n}\right) \leq H_{\lambda}(u) \leq C_{\lambda}+\varepsilon_{0}\right\} .
$$


Clearly, $F_{\varepsilon_{0}}^{*}(\lambda) \subset F_{\varepsilon_{0}}(\lambda)$. Now, we consider

$$
F^{*}(\lambda):=\left\{u \in E: H_{\lambda}(u)<C_{\lambda}-\left(\lambda-\lambda_{n}\right)\right\}
$$

and $F_{\varepsilon_{0}}^{*}(\lambda) \cup F^{*}(\lambda)$. Since $\left\|H_{\lambda}^{\prime}(u)\right\| \geq \varepsilon_{0}$ for $u \in F_{\varepsilon_{0}}^{*}(\lambda)$, we let

$$
h_{\lambda}(u):=\frac{2 H_{\lambda}^{\prime}(u)}{\left\|H_{\lambda}^{\prime}(u)\right\|^{2}} \quad \text { for } u \in F_{\varepsilon_{0}}^{*}(\lambda) .
$$

Then $\left\langle H_{\lambda}^{\prime}(u), h_{\lambda}(u)\right\rangle=2$ for $u \in F_{\varepsilon_{0}}^{*}(\lambda)$. Since $H_{\lambda}^{\prime}$ is weakly sequentially continuous, if $\left\{u_{n}\right\}$ is $\|\cdot\|$-bounded and $u_{n} \stackrel{|\cdot|_{w}}{\rightarrow} \bar{u}$, then $u_{n} \rightarrow \bar{u}$ in $E$, hence

$$
\left\langle H_{\lambda}^{\prime}\left(u_{n}\right), h_{\lambda}(u)\right\rangle \rightarrow\left\langle H_{\lambda}^{\prime}(\bar{u}), h_{\lambda}(u)\right\rangle
$$

as $n \rightarrow \infty$. It follows that $\left\langle H_{\lambda}^{\prime}(\cdot), h_{\lambda}(u)\right\rangle$ is $|\cdot|_{w}$-continuous on sets bounded in $E$. Therefore, there is an open $\mid \cdot{ }_{w}$-neighborhood $\mathcal{N}_{u}$ of $u$ such that

$$
\left\langle H_{\lambda}^{\prime}(v), h_{\lambda}(u)\right\rangle>1 \quad \text { for } v \in \mathcal{N}_{u}, u \in F_{\varepsilon_{0}}^{*}(\lambda)
$$

On the other hand, since $H_{\lambda}$ is $|\cdot|_{w}$-upper semi-continuous, $F^{*}(\lambda)$ is $|\cdot|_{w}$-open. Consequently,

$$
\mathcal{N}_{\lambda}:=\left\{\mathcal{N}_{u}: u \in F_{\varepsilon_{0}}^{*}(\lambda)\right\} \cup F^{*}(\lambda)
$$

is an open cover of $F_{\varepsilon_{0}}^{*}(\lambda) \cup F^{*}(\lambda)$. Now we may find a $|\cdot|_{w}$-locally finite and $|\cdot|_{w}$ open refinement $\left(\mathcal{U}_{j}\right)_{j \in J}$ with a corresponding $|\cdot|_{w}$-Lipschitz continuous partition of unity $\left(\beta_{j}\right)_{j \in J}$. For each $j$, we can either find $u_{j} \in F_{\varepsilon_{0}}^{*}(\lambda)$ such that $\mathcal{U}_{j} \subset \mathcal{N}_{u_{j}}$, or if such $u$ does not exist, then we have $\mathcal{U}_{j} \subset F^{*}(\lambda)$. In the first case we set $w_{j}(u)=h_{\lambda}\left(u_{j}\right)$; in the second case, $w_{j}(u)=0$. Let $U^{*}=\cup_{j \in J} \mathcal{U}_{j}$, then $U^{*}$ is $|\cdot|_{w}$-open and $F_{\varepsilon_{0}}^{*}(\lambda) \cup F^{*}(\lambda) \subset U^{*}$. Define

$$
Y_{\lambda}(u):=\sum_{j \in J} \beta_{j}(u) w_{j}(u)
$$

then $Y_{\lambda}: U^{*} \mapsto E$ is a vector field which has the following properties:

(1) $Y_{\lambda}$ is locally Lipschitz continuous in both $\|\cdot\|$ and $|\cdot|_{w}$ topology;

(2) $\left\langle H_{\lambda}^{\prime}(u), Y_{\lambda}(u)\right\rangle \geq 0, \forall u \in U^{*}$;

(3) $\left\langle H_{\lambda}^{\prime}(u), Y_{\lambda}(u)\right\rangle \geq 1, \forall u \in F_{\varepsilon_{0}}^{*}(\lambda)$;

(4) $\left|Y_{\lambda}(u)\right|_{w} \leq\left\|Y_{\lambda}(u)\right\| \leq 2 / \varepsilon_{0}$ for $u \in U^{*}$ and all $\lambda \in[1,2]$.

Consider the following initial value problem

$$
\frac{\mathrm{d} \eta(t, u)}{\mathrm{d} t}=-Y_{\lambda}(\eta), \quad \eta(0, u)=u
$$

for all $u \in F^{*}(\lambda) \cup F\left(\lambda, \varepsilon_{0}\right)$, where $F^{*}(\lambda)$ is given by (2.5) and

$$
F\left(\lambda, \varepsilon_{0}\right):=\left\{u \in E:\|u\| \leq k, C_{\lambda}-\left(\lambda-\lambda_{n}\right) \leq H_{\lambda}(u) \leq C_{\lambda}+\varepsilon_{0}\right\} \subset F_{\varepsilon_{0}}^{*}(\lambda) .
$$

Then by classical theory of ordinary differential equations and the properties of $Y_{\lambda}$, for each $u$ as above, there exists a unique solution $\eta(t, u)$ as long as it does not approach the boundary of $U^{*}$. Furthermore, $t \mapsto H_{\lambda}(\eta(t, u))$ is nonincreasing. 


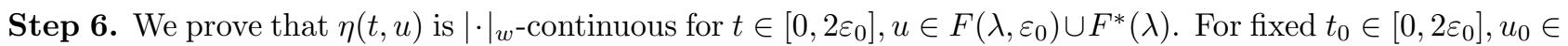
$F\left(\lambda, \varepsilon_{0}\right) \cup F^{*}(\lambda)$, we see that

$$
\eta(t, u)-\eta\left(t, u_{0}\right)=u-u_{0}+\int_{0}^{t}\left(Y_{\lambda}\left(\eta\left(s, u_{0}\right)\right)-Y_{\lambda}(\eta(s, u))\right) \mathrm{d} s
$$

Since the set $\Lambda:=\eta\left(\left[0,2 \varepsilon_{0}\right] \times\left\{u_{0}\right\}\right)$ is compact and $|\cdot|_{w}$-compact and $Y_{\lambda}$ is $|\cdot|_{w}$-locally $|\cdot|{ }_{w}$-Lipschitz, there exist $r_{1}>0, r_{2}>0$ such that $\left\{u \in E: \inf _{e \in \Lambda}|u-e|_{w}<r_{1}\right\} \subset U^{*}$ and $\left|Y_{\lambda}(u)-Y_{\lambda}(v)\right|_{w} \leq r_{2}|u-v|_{w}$ for any $u, v \in \Lambda$. Suppose that $\eta(s, u) \in U^{*}$ for $0 \leq s \leq t$. Then by $(2.8)$,

$$
\begin{aligned}
\left|\eta(t, u)-\eta\left(t, u_{0}\right)\right|_{w} & \leq\left|u-u_{0}\right|_{w}+\int_{0}^{t}\left|Y_{\lambda}\left(\eta\left(s, u_{0}\right)\right)-Y_{\lambda}(\eta(s, u))\right|_{w} \mathrm{~d} s \\
& \leq\left|u-u_{0}\right|_{w}+r_{2} \int_{0}^{t}\left|\eta\left(s, u_{0}\right)-\eta(s, u)\right|_{w} \mathrm{~d} s
\end{aligned}
$$

By the Gronwall inequality (see e.g., Lem. 6.9 of [26]),

$$
\left|\eta(t, u)-\eta\left(t, u_{0}\right)\right|_{w} \leq\left|u-u_{0}\right|_{w} \mathrm{e}^{r_{2} t} \leq\left|u-u_{0}\right|_{w} \mathrm{e}^{r_{2}}
$$

If $\left|u-u_{0}\right|_{w}<\delta$, where $0<\delta<r_{1} e^{-r_{2}}$, then $\left|\eta(t, u)-\eta\left(t, u_{0}\right)\right|_{w}<r_{1}$. Therefore, if $\left|t-t_{0}\right|<\delta$,

$$
\begin{aligned}
\left|\eta(t, u)-\eta\left(t_{0}, u_{0}\right)\right|_{w} & \leq\left|\eta(t, u)-\eta\left(t, u_{0}\right)\right|_{w}+\left|\eta\left(t, u_{0}\right)-\eta\left(t_{0}, u_{0}\right)\right|_{w} \\
& \leq\left|\eta(t, u)-\eta\left(t, u_{0}\right)\right|_{w}+\left|\int_{t_{0}}^{t} Y_{\lambda}\left(\eta\left(s, u_{0}\right)\right) \mathrm{d} s\right|_{w} \\
& \leq \delta \mathrm{e}^{r_{2}}+\delta c \\
& \rightarrow 0 \quad \text { as } \delta \rightarrow 0 .
\end{aligned}
$$

Step 7. Consider

$$
\eta^{*}(t, u)= \begin{cases}h_{n}(2 t, u) & 0 \leq t \leq 1 / 2 \\ \eta\left(4 \varepsilon_{0} t-2 \varepsilon_{0}, h_{n}(1, u)\right) & 1 / 2 \leq t \leq 1\end{cases}
$$

We prove that $\eta^{*} \in \Gamma$.

Evidently, for $u \in \bar{Q}$, we have either $h_{n}(1, u) \in F^{*}(\lambda)$ or $C_{\lambda}-\left(\lambda-\lambda_{n}\right) \leq H_{\lambda}\left(h_{n}(1, u)\right)$. For the later case, we observe that $\left\|h_{n}(1, u)\right\| \leq k$ by Step 3 and $H_{\lambda}\left(h_{n}(1, u)\right) \leq C_{\lambda}+\varepsilon_{0}$ by Step 4 , hence, $h_{n}(1, u) \in F\left(\lambda, \varepsilon_{0}\right)$. In view of Step $6, \eta^{*}$ is $|\cdot|_{w^{-}}$continuous satisfying $\eta^{*}(0, u)=u$ and $H\left(\eta^{*}(t, u)\right) \leq H(u)$. Now for any $\left(s_{0}, u_{0}\right) \in[0,1] \times \bar{Q}$, since $h_{n} \in \Gamma$, we first find a $|\cdot|_{w}$-neighborhood $U_{\left(s_{0}, u_{0}\right)}^{1}$ such that

$$
\left\{u-h_{n}(s, u):(s, u) \in U_{\left(s_{0}, u_{0}\right)}^{1} \cap([0,1] \times \bar{Q})\right\} \subset E_{\mathrm{fin}} .
$$

Furthermore, it is easy to see that there exists a $|\cdot|_{w}$-neighborhood $U_{\left(s_{0}, u_{0}\right)}^{2}$ of $\left(s_{0}, u_{0}\right)$ such that

$$
\left\{h_{n}(s, u)-h_{n}(2 s, u):(s, u) \in U_{\left(s_{0}, u_{0}\right)}^{2} \cap([0,1] \times \bar{Q})\right\} \subset E_{\mathrm{fin}} .
$$

Next, we have to estimate $h_{n}(t, u)-\eta\left(4 \varepsilon_{0} t-2 \varepsilon_{0}, h_{n}(1, u)\right)$ for $t \in[1 / 2,1]$. If $H_{\lambda}\left(h_{n}\left(1, u_{0}\right)\right)<C_{\lambda}-\left(\lambda-\lambda_{n}\right)$, then

$$
H_{\lambda}\left(\eta\left(t, h_{n}\left(1, u_{0}\right)\right)\right) \leq H_{\lambda}\left(h_{n}\left(1, u_{0}\right)\right)<C_{\lambda}-\left(\lambda-\lambda_{n}\right), \quad \text { for } t \in\left[0,2 \varepsilon_{0}\right]
$$

Particularly, $\eta\left(t, h_{n}\left(1, u_{0}\right)\right) \in F^{*}(\lambda)($ see $(2.5))$. 
If $H_{\lambda}\left(h_{n}\left(1, u_{0}\right)\right) \geq C_{\lambda}-\left(\lambda-\lambda_{n}\right)$, then by Step $3,\left\|h_{n}\left(1, u_{0}\right)\right\| \leq k$ and by Step 4 ,

$$
h_{n}\left(1, u_{0}\right) \in F\left(\lambda, \varepsilon_{0}\right) \subset F_{\varepsilon_{0}}^{*}(\lambda) .
$$

Since

$$
\begin{aligned}
\left\|\eta\left(t, h_{n}\left(1, u_{0}\right)\right)-h_{n}\left(1, u_{0}\right)\right\| & =\left\|\int_{0}^{t} \mathrm{~d} \eta\left(s, h_{n}\left(1, u_{0}\right)\right)\right\| \\
& \leq \int_{0}^{t}\left\|Y_{\lambda}\left(\eta\left(s, h_{n}\left(1, u_{0}\right)\right)\right)\right\| \mathrm{d} s \\
& \leq \frac{2 t}{\varepsilon_{0}},
\end{aligned}
$$

hence

$$
\left\|\eta\left(t, h_{n}\left(1, u_{0}\right)\right)\right\| \leq\left\|h_{n}\left(1, u_{0}\right)\right\|+\frac{2 t}{\varepsilon_{0}} \leq k+4, \quad \text { for } t \in\left[0,2 \varepsilon_{0}\right]
$$

Further, by Step $4, H_{\lambda}\left(\eta\left(t, h_{n}\left(1, u_{0}\right)\right) \leq H_{\lambda}\left(h_{n}\left(1, u_{0}\right)\right) \leq C_{\lambda}+\varepsilon_{0}\right.$. Therefore, for this case,

$$
\eta\left(t, h_{n}\left(1, u_{0}\right)\right) \in F_{\varepsilon_{0}}^{*}(\lambda) \cup F^{*}(\lambda), \quad t \in\left[0,2 \varepsilon_{0}\right] .
$$

Consider $\Lambda_{1}:=\left\{\eta\left(\left[0,2 \varepsilon_{0}\right], h_{n}\left(1, u_{0}\right)\right)\right\}$, which is $|\cdot|_{w}$-compact and contained in $U^{*}$ of Step 5 because of (2.11) and (2.14). Moreover, there are $r_{3}>0, r_{4}>0$ such that

- $\Lambda_{2}:=\left\{u \in E:\left|u-\Lambda_{1}\right|_{w}<r_{3}\right\} \subset U^{*}$;

- $\left|Y_{\lambda}(u)-Y_{\lambda}(v)\right|_{w} \leq r_{4}|u-v|_{w}, \quad \forall u, v \in \Lambda_{2}$;

- $Y_{\lambda}\left(\Lambda_{2}\right) \subset E_{\text {fin }}$.

Evidently, by the $|\cdot|_{w}$ continuity of $Y_{\lambda}, \eta$, and $h_{n}$, there exists a $|\cdot|_{w}$-neighborhood $U_{\left(s_{0}, u_{0}\right)}^{3}$ such that

$$
\eta\left(t, h_{n}(1, u)\right) \subset \Lambda_{2}
$$

for $t \in\left[0,2 \varepsilon_{0}\right]$ and $u \in U_{\left(s_{0}, u_{0}\right)}^{3}$. For $t \in[1 / 2,1]$, note that

$$
\begin{aligned}
& h_{n}(t, u)-\eta\left(4 \varepsilon_{0} t-2 \varepsilon_{0}, h_{n}(1, u)\right) \\
& =h_{n}(t, u)-h_{n}(1, u)+\int_{0}^{4 \varepsilon_{0} t-2 \varepsilon_{0}} Y_{\lambda}\left(\eta\left(s, h_{n}(1, u)\right)\right) \mathrm{d} s
\end{aligned}
$$

we conclude by (2.15) that

$$
\left\{h_{n}(t, u)-\eta\left(4 \varepsilon_{0} t-2 \varepsilon_{0}, h_{n}(1, u)\right):(t, u) \in U_{\left(s_{0}, u_{0}\right)}^{3} \cap([1 / 2,1] \times \bar{Q})\right\} \subset E_{\mathrm{fin}} .
$$

According to the definition of $\eta^{*}$,

$$
\begin{gathered}
u-\eta^{*}(t, u)=u-h_{n}(t, u)+h_{n}(t, u)-h_{n}(2 t, u), \quad t \in[0,1 / 2] ; \\
u-\eta^{*}(t, u)=u-h_{n}(t, u)+h_{n}(t, u)-\eta\left(4 \varepsilon_{0} t-2 \varepsilon_{0}, h_{n}(1, u)\right), \quad t \in[1 / 2,1] .
\end{gathered}
$$

Therefore, by combining $(2.9,2.10)$ and $(2.16)$, we obtain that

$$
\left\{u-\eta^{*}(t, u):(t, u) \in \tilde{U}_{\left(s_{0}, u_{0}\right)}^{*} \cap([0,1] \times \bar{Q})\right\} \subset E_{\mathrm{fin}},
$$

which implies that $\eta^{*} \in \Gamma$, where $\tilde{U}_{\left(s_{0}, u_{0}\right)}^{*}=U_{\left(s_{0}, u_{0}\right)}^{1} \cap U_{\left(s_{0}, u_{0}\right)}^{2}$ or $\tilde{U}_{\left(s_{0}, u_{0}\right)}^{*}=U_{\left(s_{0}, u_{0}\right)}^{1} \cap U_{\left(s_{0}, u_{0}\right)}^{3}$ 
Step 8. We will get a contradiction in this step.

Case 1: if $H_{\lambda}\left(h_{n}(1, u)\right)<C_{\lambda}-\left(\lambda-\lambda_{n}\right)$ for some $u \in \bar{Q}$, then $h_{n}(1, u) \in F^{*}(\lambda)$ (see $\left.(2.5)\right)$ and

$$
H_{\lambda}\left(\eta^{*}(1, u)\right)=H_{\lambda}\left(\eta\left(2 \varepsilon_{0}, h_{n}(1, u)\right) \leq H_{\lambda}\left(h_{n}(1, u)\right)\right)<C_{\lambda}-\left(\lambda-\lambda_{n}\right) .
$$

Case 2: if $H_{\lambda}\left(h_{n}(1, u)\right) \geq C_{\lambda}-\left(\lambda-\lambda_{n}\right)$ for some $u \in \bar{Q}$, then by Step 3 and Step $4,\left\|h_{n}(1, u)\right\| \leq k$ and $\sup _{u \in \bar{Q}} H_{\lambda}\left(h_{n}(1, u)\right) \leq C_{\lambda}+\varepsilon_{0}$. Then, $h_{n}(1, u) \in F_{\varepsilon_{0}}^{*}(\lambda)$. Assume that $H_{\lambda}\left(\eta^{*}(1, u)\right) \geq C_{\lambda}-\left(\lambda-\lambda_{n}\right)$, then for $0 \leq t \leq 2 \varepsilon_{0}$, we have,

$$
\begin{aligned}
C_{\lambda}-\left(\lambda-\lambda_{n}\right) & \leq H_{\lambda}\left(\eta^{*}(1, u)\right) \\
& =H_{\lambda}\left(\eta\left(2 \varepsilon_{0}, h_{n}(1, u)\right)\right) \\
& \leq H_{\lambda}\left(\eta\left(t, h_{n}(1, u)\right)\right) \\
& \leq H_{\lambda}\left(\eta\left(0, h_{n}(1, u)\right)\right) \\
& =H_{\lambda}\left(h_{n}(1, u)\right) \\
& \leq C_{\lambda}+\varepsilon_{0} .
\end{aligned}
$$

Furthermore, for any $t \in\left[0,2 \varepsilon_{0}\right]$, by Property (4) of $Y_{\lambda}$ (see (2.6)),

$$
\begin{aligned}
\left\|\eta\left(t, h_{n}(1, u)\right)-h_{n}(1, u)\right\| & =\left\|\int_{0}^{t} \frac{\mathrm{d} \eta\left(s, h_{n}(1, u)\right)}{\mathrm{d} s} \mathrm{~d} s\right\| \\
& \leq \int_{0}^{t} \| Y_{\lambda}\left(\eta\left(s, h_{n}(1, u)\right) \| \mathrm{d} s\right. \\
& \leq 2 t / \varepsilon_{0},
\end{aligned}
$$

it follows that

$$
\left\|\eta\left(t, h_{n}(1, u)\right)\right\| \leq 2 t / \varepsilon_{0}+\left\|h_{n}(1, u)\right\| \leq k+4 \quad \text { for } t \in\left[0,2 \varepsilon_{0}\right]
$$

Hence, equations (2.18) and (2.19) imply that $\eta\left(t, h_{n}(1, u)\right) \in F_{\varepsilon_{0}}^{*}(\lambda)$ for $t \in\left[0,2 \varepsilon_{0}\right]$. Since on $F_{\varepsilon_{0}}^{*}(\lambda)$, $\left\langle H_{\lambda}^{\prime}(u), Y_{\lambda}(u)\right\rangle>1$, then

$$
\begin{aligned}
\left.H_{\lambda}\left(\eta\left(2 \varepsilon_{0}, h_{n}(1, u)\right)\right)-H_{\lambda}\left(h_{n}(1, u)\right)\right) & =\int_{0}^{2 \varepsilon_{0}} \frac{\mathrm{d}}{\mathrm{d} t} H_{\lambda}\left(\eta\left(t, h_{n}(1, u)\right)\right) \mathrm{d} t \\
& =-\int_{0}^{2 \varepsilon_{0}}\left\langle H_{\lambda}^{\prime}\left(\eta\left(t, h_{n}(1, u)\right)\right), Y_{\lambda}\left(\eta\left(t, h_{n}(1, u)\right)\right)\right\rangle \mathrm{d} t \\
& \leq-2 \varepsilon_{0} .
\end{aligned}
$$

Therefore, by Step 4,

$$
\begin{aligned}
H_{\lambda}\left(\eta\left(2 \varepsilon_{0}, h_{n}(1, u)\right)\right) & \leq H_{\lambda}\left(h_{n}(1, u)\right)-2 \varepsilon_{0} \\
& \leq C_{\lambda}-\varepsilon_{0} \\
& \leq C_{\lambda}-\left(\lambda-\lambda_{n}\right) .
\end{aligned}
$$

Combining (2.17) and (2.20), we find

$$
H_{\lambda}\left(\eta^{*}(1, u)\right)=H_{\lambda}\left(\eta\left(2 \varepsilon_{0}, h_{n}(1, u)\right)\right) \leq C_{\lambda}-\left(\lambda-\lambda_{n}\right)
$$

for any $(t, u) \in[0,1] \times \bar{Q}$, which contradicts the definition of $C_{\lambda}$. 


\section{SCHRÖDINGER EQUATION}

Let $E:=W^{1,2}\left(\mathbf{R}^{N}\right)$. It is well known that there is a one-to-one correspondence between solutions of $(\mathrm{S})$ and critical points of the $\mathcal{C}^{1}(E, \mathbf{R})$-functional

$$
H(u):=\frac{1}{2} \int_{\mathbf{R}^{N}}\left(|\nabla u|^{2}+V(x) u^{2}\right) \mathrm{d} x-\frac{1}{2^{*}} \int_{\mathbf{R}^{N}} K(x)|u|^{2^{*}} \mathrm{~d} x-\int_{\mathbf{R}^{N}} G(x, u) \mathrm{d} x .
$$

Let $(E(\lambda))_{\lambda \in \mathbf{R}}$ be the spectral family of $-\Delta+V$ in $L^{2}\left(\mathbf{R}^{N}\right)$. Let $E^{-}:=E(0) L^{2} \cap E$ and $E^{+}:=(i d-E(0)) L^{2} \cap E$, then the quadratic form $\int_{\mathbf{R}^{N}}\left(|\nabla u|^{2}+V u^{2}\right) \mathrm{d} x$ is positive definite on $E^{+}$and negative definite on $E^{-}(c f$. [22]). By introducing a new inner product $\langle\cdot, \cdot\rangle$ in $E$, the corresponding norm $\|\cdot\|$ is equivalent to $\|\cdot\|_{1,2}$, the usual norm of $W^{1,2}\left(\mathbf{R}^{N}\right)$. Moreover, $\int_{\mathbf{R}^{N}}\left(|\nabla u|^{2}+V u^{2}\right) \mathrm{d} x=\left\|u^{+}\right\|^{2}-\left\|u^{-}\right\|^{2}$, where $u^{ \pm} \in E^{ \pm}$. Then functional (3.1) can be rewritten as

$$
H(u)=\frac{1}{2}\left\|u^{+}\right\|^{2}-\frac{1}{2}\left\|u^{-}\right\|^{2}-\frac{1}{2^{*}} \int_{\mathbf{R}^{N}} K(x)|u|^{2^{*}} \mathrm{~d} x-\int_{\mathbf{R}^{N}} G(x, u) \mathrm{d} x .
$$

In order to use Theorem 2.1, we consider the family of functional defined by

$$
H_{\lambda}(u)=\frac{1}{2}\left\|u^{+}\right\|^{2}-\lambda\left(\frac{1}{2}\left\|u^{-}\right\|^{2}+\frac{1}{2^{*}} \int_{\mathbf{R}^{N}} K(x)|u|^{2^{*}} \mathrm{~d} x+\int_{\mathbf{R}^{N}} G(x, u) \mathrm{d} x\right)
$$

for $\lambda \in[1,2]$.

Lemma 3.1. $H_{\lambda}$ is $|\cdot|_{w}$-upper semicontinuous. $H_{\lambda}^{\prime}$ is weakly sequentially continuous.

Proof. Noting that $u_{n}:=u_{n}^{-}+u_{n}^{+} \stackrel{|\cdot|_{w}}{\rightarrow} u$ implies that $u_{n} \rightarrow u$ weakly in $E$ and $u_{n}^{+} \rightarrow u^{+}$strongly in $E$, then the proof is the same as that in $[23]$ (see also $[6,12]$ ). The second conclusion is due to [6].

Let

$$
\varphi_{\varepsilon}(x):=\frac{c_{N} \psi(x) \varepsilon^{(N-2) / 2}}{\left(\varepsilon^{2}+|x|^{2}\right)^{(N-2) / 2}}
$$

where $c_{N}=(N(N-2))^{(N-2) / 4}, \varepsilon>0$ and $\psi \in \mathcal{C}_{0}^{\infty}\left(\mathbf{R}^{N},[0,1]\right)$ with $\psi(x)=1$ if $|x| \leq r / 2 ; \psi(x)=0$ if $|x| \geq r, r$ small enough (cf. e.g. pp. 35 and 52 of [26]). Write $\varphi_{\varepsilon}=\varphi_{\varepsilon}^{+}+\varphi_{\varepsilon}^{-}$with $\varphi_{\varepsilon}^{+} \in E^{+}, \varphi_{\varepsilon}^{-} \in E^{-}$. Then

$$
\left\|\varphi_{\varepsilon}^{-}\right\| \rightarrow 0,\left\|\varphi_{\varepsilon}^{+}\right\|_{2^{*}}^{2^{*}} \rightarrow S^{N / 2} \quad \text { as } \varepsilon \rightarrow 0(c f . \text { Prop. } 4.2 \text { of }[6])
$$

where

$$
S:=\inf _{u \in E \backslash\{0\}} \frac{\|\nabla u\|_{2}^{2}}{\|u\|_{2^{*}}^{2}} .
$$

The following lemma can be found in Proposition 4.2 of [6].

Lemma 3.2. Set

then

$$
I_{1}(u):=\frac{1}{2}\left\|u^{+}\right\|^{2}-\frac{1}{2}\left\|u^{-}\right\|^{2}-\frac{1}{2^{*}} \int_{\mathbf{R}^{N}} K(x)|u|^{2^{*}} \mathrm{~d} x, \quad u \in E,
$$

for $\varepsilon$ small enough, where $Z_{\varepsilon}:=E^{-} \oplus \mathbf{R} \varphi_{\varepsilon}^{+}$.

$$
\sup _{Z_{\varepsilon}} I_{1}<c^{*}:=\frac{S^{N / 2}}{N\|K\|_{\infty}^{(N-2) / 2}},
$$

To carry forward, we prepare an auxiliary results. 
Lemma 3.3. Assume that $g(x, u) / u \rightarrow 0$ as $|u| \rightarrow 0$ uniformly for $x \in \mathbf{R}^{N}$ and that $g$ is of subcritical Sobolev exponent growth. If a bounded sequence $\left(w_{n}\right) \subset E$ and $\lambda_{n} \in[1,2]$ satisfy

$$
\lambda_{n} \rightarrow \lambda, \quad H_{\lambda_{n}}^{\prime}\left(w_{n}\right) \rightarrow 0, \quad H_{\lambda_{n}}\left(w_{n}\right) \rightarrow c(\lambda)
$$

where $0<c(\lambda)<c_{\lambda}^{*}:=\frac{S^{N / 2}}{N\|\lambda K\|_{\infty}^{(N-2) / 2}}$, then $\left(w_{n}\right)$ is nonvanishing, i.e., there exist $r, \eta>0$ and a sequence $\left(y_{n}\right) \subset \mathbf{R}^{N}$, a sequence of open ball $\left(B\left(y_{n}, r\right)\right)$ centered at $y_{n}$ with radius $r$, such that

$$
\limsup _{n \rightarrow \infty} \int_{B\left(y_{n}, r\right)} w_{n}^{2} \mathrm{~d} x \geq \eta
$$

Proof. The idea is essentially due to Proposition 4.1 of [6]. We give the sketch for the reader's convenience.

If $\left(w_{n}\right)$ is not nonvanishing, then $w_{n} \rightarrow 0$ in $L^{r}\left(\mathbf{R}^{N}\right)$ for $2<r<2^{*}$ by Lions' lemma ([16], Lem 1.21). By standard arguments,

$$
\int_{\mathbf{R}^{N}} g\left(x, w_{n}\right) v_{n} \mathrm{~d} x \rightarrow 0 \text { and } \int_{\mathbf{R}^{N}} G\left(x, w_{n}\right) \mathrm{d} x \rightarrow 0
$$

whenever $\left(v_{n}\right) \subset E$ is bounded. Hence

$$
H_{\lambda_{n}}\left(w_{n}\right)-\frac{1}{2}\left\langle H_{\lambda_{n}}^{\prime}\left(w_{n}\right), w_{n}\right\rangle=\frac{\lambda_{n}}{N} \int_{\mathbf{R}^{N}} K(x)\left|w_{n}\right|^{2^{*}} \mathrm{~d} x+o(1) \rightarrow c(\lambda)
$$

For any $\delta>0$, we choose $\mu>\|V\|_{\infty}(1+\delta) / \delta$. Write $w_{n}=w_{n}^{+}+w_{n}^{-} \in E^{+} \oplus E^{-}$, and let $w_{n}^{+}=\tilde{w}_{n}+\tilde{z}_{n}$, with $\tilde{w}_{n} \in E(\mu) L^{2}, \tilde{z}_{n} \in(i d-E(\mu)) L^{2}$, where $(E(\lambda))_{\lambda \in \mathbf{R}}$ is the spectral family of $-\Delta+V$ in $L^{2}$. By Proposition 2.4 of $[6], \tilde{w}_{n} \in E$ and

$$
\left\|w_{n}^{-}\right\|_{q} \leq c\left\|w_{n}^{-}\right\|_{2} \leq c\left\|w_{n}\right\| \quad \text { and } \quad\left\|\tilde{w}_{n}\right\|_{q} \leq c\left\|\tilde{w}_{n}\right\|_{2} \leq c\left\|w_{n}\right\|
$$

where $q=2 N /(N-4)$ if $N>4$ and $q$ may be chosen arbitrarily large if $N=4$. Therefore,

$$
\begin{aligned}
\lambda_{n}\left\|w_{n}^{-}\right\|^{2} & =-\left\langle H_{\lambda_{n}}^{\prime}\left(w_{n}\right), w_{n}^{-}\right\rangle-\lambda_{n} \int_{\mathbf{R}^{N}} K(x)\left|w_{n}\right|^{2^{*}-2} w_{n} w_{n}^{-} \mathrm{d} x-\lambda_{n} \int_{\mathbf{R}^{N}} g\left(x, w_{n}\right) w_{n}^{-} \mathrm{d} x \\
& \leq 2\|K\|_{\infty}\left\|w_{n}\right\|_{r}^{2^{*}-1}\left\|w_{n}^{-}\right\|_{q}+o(1) \\
& \rightarrow 0
\end{aligned}
$$

where $r$ satisfies $\left(2^{*}-1\right) / r+1 / q=1$, hence $2<r<2^{*}$. By the same reasoning,

$$
\left\|\tilde{w}_{n}\right\| \rightarrow 0, \quad \text { hence, } \quad w_{n}-\tilde{z}_{n} \rightarrow 0 \text {. }
$$

It follows that

$$
\begin{aligned}
\left\|\tilde{z}_{n}\right\|^{2} & =\int_{\mathbf{R}^{N}}\left(\left|\nabla \tilde{z}_{n}\right|^{2}+V \tilde{z}_{n}^{2}\right) \mathrm{d} x \\
& =\lambda_{n} \int_{\mathbf{R}^{N}} K(x)\left|w_{n}\right|^{2^{*}-2} w_{n} \tilde{z}_{n} \mathrm{~d} x+o(1) \\
& =\lambda_{n} \int_{\mathbf{R}^{N}} K(x)\left|w_{n}\right|^{2^{*}} \mathrm{~d} x
\end{aligned}
$$

On the other hand, by (4.6) of [6], for any $\delta>0$ and $\mu>\|V\|_{\infty}(1+\delta) / \delta$, we have that

$$
(1-\delta) \int_{\mathbf{R}^{N}}\left|\nabla \tilde{z}_{n}\right|^{2} \mathrm{~d} x \leq \int_{\mathbf{R}^{N}}\left(\left|\nabla \tilde{z}_{n}\right|^{2}+V \tilde{z}_{n}^{2}\right) \mathrm{d} x
$$


By $(3.9,3.8)$ and $(3.10)$, we have that

$$
\begin{aligned}
\left(\lambda \int_{\mathbf{R}^{N}} K(x)\left|w_{n}\right|^{2^{*}} \mathrm{~d} x\right)^{2 / 2^{*}} & \leq\left(\lambda\|K\|_{\infty}\right)^{2 / 2^{*}}\left\|w_{n}\right\|_{2^{*}}^{2} \\
& =\left(\lambda\|K\|_{\infty}\right)^{2 / 2^{*}}\left\|\tilde{z}_{n}\right\|_{2^{*}}^{2}+o(1) \\
& \leq\left(\lambda\|K\|_{\infty}\right)^{2 / 2^{*}}\left\|\nabla \tilde{z}_{n}\right\|_{2}^{2} / S+o(1) \\
& \leq \frac{\left(\lambda\|K\|_{\infty}\right)^{2 / 2^{*}}}{S(1-\delta)} \lambda \int_{\mathbf{R}^{N}} K(x)\left|w_{n}\right|^{2^{*}} \mathrm{~d} x+o(1) .
\end{aligned}
$$

If we let $n \rightarrow \infty$ and use (3.6), it follows that

$$
(N c(\lambda))^{2 / 2^{*}} \leq \frac{\left(\lambda\|K\|_{\infty}\right)^{2 / 2^{*}}}{S(1-\delta)} N c(\lambda)
$$

which implies that either $c(\lambda)=0$ or $c(\lambda) \geq(1-\delta)^{N / 2} c_{\lambda}^{*}$. Either way, we get a contradiction since $\delta$ is chosen arbitrarily.

Choose $z_{0}:=\varphi_{\varepsilon}^{+} /\left\|\varphi_{\varepsilon}^{+}\right\| \in E^{+}$. For $R>0$, set $Q:=\left\{u=u^{-}+s z_{0}:\|u\|<R, u^{-} \in E^{-}, s \in \mathbf{R}^{+}\right\}$. Let $p_{0}=s_{0} z_{0} \in Q, s_{0}>0$. For any $u \in E$, we write $u=u^{-}+s z_{0}+w$ with $u^{-} \in E^{-}, w \in\left(E^{-} \oplus \mathbf{R} z_{0}\right)^{\perp}, s \in \mathbf{R}$. Consider a map $F: E \rightarrow E^{-} \oplus \mathbf{R} z_{0}$ defined by

$$
F\left(u^{-}+s z_{0}+w\right)=u^{-}+\left\|s z_{0}+w\right\| z_{0}
$$

Let $B:=F^{-1}\left(p_{0}\right)$, then

$$
B=\left\{u=s z_{0}+w: w \in\left(E^{-} \oplus \mathbf{R} z_{0}\right)^{\perp},\|u\|=s_{0}\right\}
$$

It is easy to check that $F, p_{0}, B$ satisfy the basic assumptions in Section 2. By hypotheses $\left(\mathrm{S}_{4}\right)$ and $\left(\mathrm{S}_{5}\right)$, the proof of the next lemma is trivial.

Lemma 3.4. There exist $R>0, s_{0}>0$, such that

$$
\inf _{B} H_{\lambda}>0, \quad \sup _{\partial \bar{Q}} H_{\lambda} \leq 0, \quad \text { for all } \lambda \in[1,2]
$$

Lemma 3.5. For almost all $\lambda \in[1,2]$, there exists $\left\{u_{n}\right\} \in E$ such that

$$
\sup _{n}\left\|u_{n}\right\|<\infty, \quad H_{\lambda}^{\prime}\left(u_{n}\right) \rightarrow 0 \quad \text { and } \quad H_{\lambda}\left(u_{n}\right) \rightarrow C_{\lambda}
$$

where $C_{\lambda} \in\left[\inf _{B} H_{\lambda}, \sup _{\bar{Q}} H\right]$. Furthermore, there exists $\delta_{0}>0$ small enough such that, for almost all $\lambda \in\left[1,1+\delta_{0}\right]$, there exists $u_{\lambda} \neq 0$ such that

$$
H_{\lambda}^{\prime}\left(u_{\lambda}\right)=0, \quad H_{\lambda}\left(u_{\lambda}\right) \leq \sup _{\bar{Q}} H
$$

Proof. The first conclusion follows immediately from Lemmas 3.1, 3.4, 3.5 and Theorem 2.1. Now we prove the second conclusion. Since $g(x, u) u \geq 0$ and $\bar{Q} \subset Z_{\varepsilon}$, we get that

$$
0<C_{\lambda} \leq \sup _{\bar{Q}} H \leq \sup _{Z_{\varepsilon}} I_{1}<c^{*}
$$

where $I_{1}, c^{*}$ and $Z_{\varepsilon}$ come from Lemma 3.2. Therefore, there exists $\delta_{0}>0$ such that $0<C_{\lambda}<c_{\lambda}^{*}$ for almost all $\lambda \in\left[1,1+\delta_{0}\right]$, where $c_{\lambda}^{*}$ comes from Lemma 3.3. For those $\lambda$, by Lemma 3.3, $\left\{u_{n}\right\}$ is nonvanishing, that is, 
there exist $y_{n} \in \mathbf{R}^{N}, \alpha>0, R_{1}>0$ such that

$$
\limsup _{n \rightarrow \infty} \int_{B\left(y_{n}, R_{1}\right)}\left|u_{n}\right|^{2} \mathrm{~d} x \geq \alpha>0 .
$$

We find $\bar{y}_{n} \in \mathbf{Z}^{N}$ such that

$$
\limsup _{n \rightarrow \infty} \int_{B\left(0,2 R_{1}\right)}\left|v_{n}\right|^{2} \mathrm{~d} x \geq \alpha>0
$$

where $v_{n}(x):=u_{n}\left(x+\bar{y}_{n}\right)$. By the periodicity of $V, K$ and $g,\left\{v_{n}\right\}$ is still bounded and

$$
\lim _{n \rightarrow \infty} H_{\lambda}\left(v_{n}\right) \in\left[\inf _{B} H_{\lambda}, \sup _{\bar{Q}} H\right], \quad \lim _{n \rightarrow \infty} H_{\lambda}^{\prime}\left(v_{n}\right)=0 .
$$

We may suppose that $v_{n} \rightarrow u_{\lambda}$. Since $E$ is embedded compactly in $L_{\text {loc }}^{t}\left(\mathbf{R}^{N}\right)$ for $2 \leq t<2^{*}$, then

$$
0<\alpha \leq \lim _{n \rightarrow \infty} \int_{B\left(0,2 R_{1}\right)}\left|v_{n}\right|^{2} \mathrm{~d} x=\int_{B\left(0,2 R_{1}\right)}\left|u_{\lambda}\right|^{2} \mathrm{~d} x \leq\left|u_{\lambda}\right|_{2}^{2}
$$

therefore, $u_{\lambda} \neq 0$. Since $H_{\lambda}^{\prime}$ is weakly sequentially continuous, $H_{\lambda}^{\prime}\left(u_{\lambda}\right)=0$. Finally, by Fatou's lemma,

$$
\begin{aligned}
H_{\lambda}\left(u_{\lambda}\right) & =H_{\lambda}\left(u_{\lambda}\right)-\frac{1}{2}\left\langle H_{\lambda}^{\prime}\left(u_{\lambda}\right), u_{\lambda}\right\rangle \\
& =\lambda \int_{\mathbf{R}^{N}}\left(\frac{1}{2}\left(K(x)\left|u_{\lambda}\right|^{2^{*}}+g\left(x, u_{\lambda}\right) u_{\lambda}\right)-\frac{1}{2^{*}} K(x)\left|u_{\lambda}\right|^{2^{*}}-G\left(x, u_{\lambda}\right)\right) \mathrm{d} x \\
& =\lambda \int_{\mathbf{R}^{N}} \lim _{n \rightarrow \infty}\left(\frac{1}{2}\left(K(x)\left|v_{n}\right|^{2^{*}}+g\left(x, v_{n}\right) v_{n}\right)-\frac{1}{2^{*}} K(x)\left|v_{n}\right|^{2^{*}}-G\left(x, v_{n}\right)\right) \mathrm{d} x \\
& \leq \lim _{n \rightarrow \infty}\left(H_{\lambda}\left(v_{n}\right)-\frac{1}{2}\left\langle H_{\lambda}^{\prime}\left(v_{n}\right), v_{n}\right\rangle\right) \\
& \leq \lim _{n \rightarrow \infty} H_{\lambda}\left(v_{n}\right) \\
& \leq \sup _{\bar{Q}} H .
\end{aligned}
$$

Lemma 3.6. There exist $\lambda_{n} \in\left[1,1+\delta_{0}\right]$ with $\lambda_{n} \rightarrow 1$, and $z_{n} \in E \backslash\{0\}$ such that

$$
H_{\lambda_{n}}^{\prime}\left(z_{n}\right)=0, \quad H_{\lambda_{n}}\left(z_{n}\right) \leq \sup _{\bar{Q}} H .
$$

Proof. It is an immediately consequence of Lemma 3.5 .

Lemma 3.7. $\left\{z_{n}\right\}$ is bounded.

Proof. Let $g_{1}(x, u):=K(x)|u|^{2^{*}-2} u+g(x, u)$ and $G_{1}(x, u):=\int_{0}^{u} g_{1}(x, s) \mathrm{d} s$. Then by the assumption $\left(\mathrm{S}_{4}\right)$, we see that

$$
\lim _{u \rightarrow 0} \frac{g_{1}(x, u) u}{G_{1}(x, u)}=2^{*} \quad \text { uniformly for } x \in \mathbf{R}^{N} .
$$

Let $\varepsilon_{1}>0$ be such that $2^{*}-\varepsilon_{1}>2$. Hence, there exists $R_{1}>0$ such that

$$
g_{1}(x, u) u \geq\left(2^{*}-\varepsilon_{1}\right) G_{1}(x, u), \quad \text { for } x \in \mathbf{R}^{N},|u| \leq R_{1} .
$$


On the other hand, since $g(x, u)$ is of subcritical growth,

$$
\lim _{u \rightarrow \infty} \frac{g_{1}(x, u) u-2 G_{1}(x, u)}{|u|^{2^{*}}}=\left(1-\frac{2}{2^{*}}\right) K(x) \geq c>0
$$

uniformly for $x \in \mathbf{R}^{N}$. Furthermore, condition (1.2) implies that

$$
0<g(x, u) u \leq \frac{2}{N-2} k_{0}|u|^{2^{*}} \quad \text { for all } x \in \mathbf{R}^{N}, u \neq 0
$$

hence

$$
g_{1}(x, u) u-2 G_{1}(x, u)>0 \quad \text { for all } x \in \mathbf{R}^{N}, u \neq 0 .
$$

Therefore (3.13) and (3.14) imply that there exists $c$ small enough, such that

$$
g_{1}(x, u) u-2 G_{1}(x, u) \geq c|u|^{2^{*}} \quad \text { for all } x \in \mathbf{R}^{N},|u| \geq R_{1} .
$$

Recall that $H_{\lambda_{n}}\left(z_{n}\right) \leq \sup _{\bar{Q}} H$ and $H_{\lambda_{n}}^{\prime}\left(z_{n}\right)=0$, then

$$
\begin{aligned}
\left(\frac{1}{2}-\frac{1}{2^{*}-\varepsilon_{1}}\right)\left(\left\|z_{n}^{+}\right\|^{2}-\lambda_{n}\left\|z_{n}^{-}\right\|^{2}\right)+\lambda_{n}\left(\frac{1}{2^{*}-\varepsilon_{1}}-\frac{1}{2^{*}}\right) & \int_{\mathbf{R}^{N}} K(x)\left|z_{n}\right|^{2^{*}} \mathrm{~d} x \\
& +\lambda_{n} \int_{\mathbf{R}^{N}}\left(\frac{1}{2^{*}-\varepsilon_{1}} g\left(x, z_{n}\right) z_{n}-G\left(x, z_{n}\right)\right) \mathrm{d} x \leq \sup _{\bar{Q}} H
\end{aligned}
$$

By $(3.12,3.14)$ and $(3.16)$,

$$
\begin{aligned}
\left(\frac{1}{2}-\frac{1}{2^{*}-\varepsilon_{1}}\right)\left(\left\|z_{n}^{+}\right\|^{2}-\lambda_{n}\left\|z_{n}^{-}\right\|^{2}\right) & \leq c+c\left(\int_{\left|z_{n}\right| \leq R_{1}}+\int_{\left|z_{n}\right| \geq R_{1}}\right)\left(G_{1}\left(x, z_{n}\right)-\frac{1}{2^{*}-\varepsilon_{1}} g_{1}\left(x, z_{n}\right) z_{n}\right) \mathrm{d} x \\
& \leq c+c \int_{\left|z_{n}\right| \geq R_{1}}\left(G_{1}\left(x, z_{n}\right)-\frac{1}{2^{*}-\varepsilon_{1}} g_{1}\left(x, z_{n}\right) z_{n}\right) \mathrm{d} x \\
& \leq c+c \int_{\left|z_{n}\right| \geq R_{1}}\left(\frac{1}{2} g_{1}\left(x, z_{n}\right) z_{n}-\frac{1}{2^{*}-\varepsilon_{1}} g_{1}\left(x, z_{n}\right) z_{n}\right) \mathrm{d} x \\
& =c+c \int_{\left|z_{n}\right| \geq R_{1}} g_{1}\left(x, z_{n}\right) z_{n} \mathrm{~d} x
\end{aligned}
$$

Since, by $\left(\mathrm{S}_{4}\right),|g(x, z) z| \leq c|z|^{2^{*}}$ for all $(x, z) \in \mathbf{R}^{N} \times \mathbf{R},(3.17)$ implies that

$$
\begin{aligned}
\left\|z_{n}^{+}\right\|^{2}-\lambda_{n}\left\|z_{n}^{-}\right\|^{2} & \leq c+c \int_{\left|z_{n}\right| \geq R_{1}} g_{1}\left(x, z_{n}\right) z_{n} \mathrm{~d} x \\
& \leq c+c \int_{\left|z_{n}\right| \geq R_{1}}\left(K(x)\left|z_{n}\right|^{2^{*}}+g\left(x, z_{n}\right) z_{n}\right) \mathrm{d} x \\
& \leq c+c \int_{\left|z_{n}\right| \geq R_{1}}\left|z_{n}\right|^{2^{*}} \mathrm{~d} x .
\end{aligned}
$$


However (3.14) and (3.15) imply that

$$
\begin{aligned}
\sup _{\bar{Q}} H & \geq H_{\lambda_{n}}\left(z_{n}\right)-\frac{1}{2}\left\langle H_{\lambda_{n}}^{\prime}\left(z_{n}\right), z_{n}\right\rangle \\
& =\int_{\mathbf{R}^{N}}\left(\frac{1}{2} g_{1}\left(x, z_{n}\right) z_{n}-G_{1}\left(x, z_{n}\right)\right) \mathrm{d} x \\
& \geq \int_{\left|z_{n}\right| \geq R_{1}}\left(\frac{1}{2} g_{1}\left(x, z_{n}\right) z_{n}-G_{1}\left(x, z_{n}\right)\right) \mathrm{d} x \\
& \geq c \int_{\left|z_{n}\right| \geq R_{1}}\left|z_{n}\right|^{2^{*}} \mathrm{~d} x .
\end{aligned}
$$

Then, combining (3.18) and (3.19), we obtain that

$$
\left\|z_{n}^{+}\right\|^{2}-\lambda_{n}\left\|z_{n}^{-}\right\|^{2} \leq c .
$$

Noting that $\left\langle H_{\lambda_{n}}^{\prime}\left(z_{n}\right), z_{n}\right\rangle=0$, we see that

$$
\begin{aligned}
\left\|z_{n}^{+}\right\|^{2}-\lambda_{n}\left\|z_{n}^{-}\right\|^{2} & =\lambda_{n} \int_{\mathbf{R}^{N}}\left(K(x)\left|z_{n}\right|^{2^{*}}+g\left(x, z_{n}\right) z_{n}\right) \mathrm{d} x \\
& \geq c \int_{\mathbf{R}^{N}}\left|z_{n}\right|^{2^{*}} \mathrm{~d} x .
\end{aligned}
$$

So, by (3.20) and (3.21), $\int_{\mathbf{R}^{N}}\left|z_{n}\right|^{2^{*}} \mathrm{~d} x \leq c$. Noting that $\left\langle H_{\lambda_{n}}^{\prime}\left(z_{n}\right), z_{n}^{+}\right\rangle=0$ and (S $\left.\mathrm{S}_{4}\right)$, we obtain, by Hölder's inequality and (3.21), that

$$
\begin{aligned}
\left\|z_{n}^{+}\right\|^{2} & =\lambda_{n} \int_{\mathbf{R}^{N}} K(x)\left|z_{n}\right|^{2^{*}-2} z_{n} z_{n}^{+} \mathrm{d} x+\lambda_{n} \int_{\mathbf{R}^{N}} g\left(x, z_{n}\right) z_{n}^{+} \mathrm{d} x \\
& \leq c \int_{\mathbf{R}^{N}}\left|z_{n}\right|^{2^{*}-1}\left|z_{n}^{+}\right| \\
& \leq c\left\|z_{n}\right\|_{2^{*}}^{2^{*-1}}\left\|z_{n}^{+}\right\|_{2^{*}} \\
& \leq c\left\|z_{n}^{+}\right\| .
\end{aligned}
$$

Therefore $\left\|z_{n}^{+}\right\| \leq c$, and hence, $\left\|z_{n}^{-}\right\| \leq c$ by (3.21).

Lemma 3.8. $\left\{z_{n}\right\}$ is nonvanishing.

Proof. Since $\left(z_{n}\right)$ is bounded, we may assume that

$$
H_{\lambda_{n}}\left(z_{n}\right) \rightarrow c_{1} \leq \sup _{\bar{Q}} H<c^{*}(c f .(3.11)) .
$$

If $\left\{z_{n}\right\}$ is not nonvanishing (i.e., is vanishing), then it follows from Lions' lemma (cf. [16], Lem. 1.21) that $z_{n} \rightarrow 0$ in $L^{r}$ whenever $2<r<2^{*}$. The assumption $\left(\mathrm{S}_{4}\right)$ implies that

$$
\int_{\mathbf{R}^{N}} g\left(x, z_{n}\right) z_{n} \mathrm{~d} x \rightarrow 0, \quad \int_{\mathbf{R}^{N}} G\left(x, z_{n}\right) \mathrm{d} x \rightarrow 0,
$$

and consequently

$$
H_{\lambda_{n}}\left(z_{n}\right)-\frac{1}{2}\left\langle H_{\lambda_{n}}^{\prime}\left(z_{n}\right), z_{n}\right\rangle=\frac{\lambda_{n}}{N} \int_{\mathbf{R}^{N}} K(x)\left|z_{n}\right|^{2^{*}} \mathrm{~d} x+o(1) \rightarrow c_{1} .
$$


Since $K(x)>0, c_{1} \geq 0$.

Case 1: If $c_{1}>0$, then by (3.22) and Lemma 3.3, $z_{n}$ is nonvanishing.

Case 2: If $c_{1}=0$, then (3.24) implies that

$$
\int_{\mathbf{R}^{N}}\left|z_{n}\right|^{2^{*}} \mathrm{~d} x \rightarrow 0
$$

Since $H_{\lambda_{n}}^{\prime}\left(z_{n}\right)=0$, for any $\varepsilon>0$, by $\left(\mathrm{S}_{4}\right)$, we have that

$$
\begin{aligned}
\left\|z_{n}^{+}\right\|^{2} & =\lambda_{n} \int_{\mathbf{R}^{N}}\left(K(x)\left|z_{n}\right|^{2^{*}-2} z_{n} z_{n}^{+}+g\left(x, z_{n}\right) z_{n}^{+}\right) \mathrm{d} x \\
& \leq c \int_{\mathbf{R}^{N}}\left|z_{n}\right|^{2^{*}-1}\left|z_{n}^{+}\right| \mathrm{d} x+\varepsilon\left\|z_{n}\right\|\left\|z_{n}^{+}\right\|+c\left\|z_{n}\right\|_{p}^{p-1}\left\|z_{n}^{+}\right\| \\
& \leq c\left\|z_{n}\right\|^{2^{*}-1}\left\|z_{n}^{+}\right\|+\varepsilon\left\|z_{n}\right\|^{2}+\varepsilon\left\|z_{n}^{+}\right\|^{2}+\left\|z_{n}^{-}+z_{n}^{+}\right\|^{p-1}\left\|z_{n}^{+}\right\| .
\end{aligned}
$$

Since $\left\|z_{n}^{-}\right\| \leq\left\|z_{n}^{+}\right\|($see $(3.21))$ and $\varepsilon$ is arbitrary,

$$
c\left\|z_{n}^{+}\right\|^{2} \leq c\left\|z_{n}^{+}\right\|^{p}+c\left\|z_{n}^{+}\right\|^{2^{*}}
$$

which implies that $\left\|z_{n}^{+}\right\| \geq c>0$. But, $H_{\lambda_{n}}^{\prime}\left(z_{n}\right)=0$, and $\left(\mathrm{S}_{4}\right)$ implies that

$$
\begin{aligned}
\left\|z_{n}^{+}\right\|^{2} & =\lambda_{n} \int_{\mathbf{R}^{N}}\left(K(x)\left|z_{n}\right|^{2^{*}-2} z_{n} z_{n}^{+}+g\left(x, z_{n}\right) z_{n}^{+}\right) \mathrm{d} x \\
& \leq c\left\|z_{n}\right\|_{2^{*}}^{2^{*}-1}\left\|z_{n}^{+}\right\|_{2^{*}}+\varepsilon c\left\|z_{n}\right\|\left\|z_{n}^{+}\right\|+c\left\|z_{n}\right\|_{p}^{p-1}\left\|z_{n}^{+}\right\| .
\end{aligned}
$$

By the vanishing of $\left\{z_{n}\right\}$ and (3.25), $\left\|z_{n}^{+}\right\| \rightarrow 0$, a contradiction. Therefore, $\left\{z_{n}\right\}$ is nonvanishing.

Proof of Theorem 1.1. Since $\left\{z_{n}\right\}$ is nonvanishing, there exist $r>0, \alpha>0$ and $y_{n} \in \mathbf{R}^{N}$ such that

$$
\limsup _{n \rightarrow \infty} \int_{B\left(y_{n}, r\right)} z_{n}^{2} \mathrm{~d} x \geq \alpha .
$$

We may assume that $y_{n} \in \mathbf{Z}^{N}$ by taking a large $r$ if necessary. Now set $\tilde{z}_{n}(x):=z_{n}\left(x+y_{n}\right)$, since $H_{\lambda}$ is invariant with respect to the translation of $x$ by elements of $\mathbf{Z}^{N}\left(\right.$ i.e., $H_{\lambda}(u(\cdot))=H_{\lambda}(u(\cdot+y))$ whenever $\left.y \in \mathbf{Z}^{N}\right),\left\|z_{n}\right\|=\left\|\tilde{z}_{n}\right\|, H_{\lambda_{n}}\left(z_{n}\right)=H_{\lambda_{n}}\left(\tilde{z}_{n}\right)$. Without loss of generality, we may suppose, up to a subsequence, that $\tilde{z}_{n} \rightarrow z^{*}$, then (3.26) implies that $z^{*} \neq 0$ and $H_{1}^{\prime}\left(z^{*}\right)=0$, i.e., $H^{\prime}\left(z^{*}\right)=0$.

\section{Appendix}

In this Appendix, we give the proof of the existence of the new norm $|\cdot|_{w}$ satisfying $|v|_{w} \leq\|v\|, \forall v \in N$ and such that the topology induced by this norm is equivalent to the weak topology of $N$ on bounded subset of $N$, more details can be found in [9].

Let $\left\{e_{k}\right\}$ be an orthonormal basis for $N$. Define

$$
|v|_{w}=\sum_{k=1}^{\infty} \frac{\left|\left(v, e_{k}\right)\right|}{2^{k}}, \quad v \in N
$$

Then $|v|_{w}$ is a norm on $N$ and satisfies $|v|_{w} \leq\|v\|, \quad v \in N$. If $v_{j} \rightarrow v$ weakly in $N$, then there is a $C>0$ such that

$$
\left\|v_{j}\right\|,\|v\| \leq C, \quad \forall j>0 .
$$


For any $\varepsilon>0$, there exist $K>0, M>0$, such that $1 / 2^{K}<\varepsilon /(4 C)$ and $\left|\left(v_{j}-v, e_{k}\right)\right|<\varepsilon / 2$ for $1 \leq k \leq K, j>M$. Therefore,

$$
\begin{aligned}
\left|v_{j}-v\right|_{w} & =\sum_{k=1}^{\infty} \frac{\left.\mid v_{j}-v, e_{k}\right) \mid}{2^{k}} \\
& \leq \sum_{k=1}^{K} \frac{\varepsilon / 2}{2^{k}}+\sum_{k=K+1}^{\infty} \frac{2 C}{2^{k}} \\
& \leq \frac{\varepsilon}{2} \sum_{k=1}^{\infty} \frac{1}{2^{k}}+\frac{2 C}{2^{K}} \sum_{k=1}^{\infty} \frac{1}{2^{k}} \\
& \leq \frac{\varepsilon}{2}+\frac{\varepsilon}{2} .
\end{aligned}
$$

Therefore, $v_{j} \rightarrow v$ weakly in $N$ implies $\left|v_{j}-v\right|_{w} \rightarrow 0$.

Conversely, let $\left\|v_{j}\right\|,\|v\| \leq C$ for all $j>0$ and $\left|v_{j}-v\right|_{w} \rightarrow 0$. Let $\varepsilon>0$ be given. If $h=\sum_{k=1}^{\infty} \alpha_{k} e_{k} \in N$, take $K$ so large that $\left\|h_{K}\right\|<\varepsilon /(4 C)$, where $h_{K}=\sum_{k=K+1}^{\infty} \alpha_{k} e_{k}$. Take $M$ so large that $\left|v_{j}-v\right|_{w}<\varepsilon /\left(2 \max _{1 \leq k \leq K} 2^{k}\left|\alpha_{k}\right|\right)$ for all $j>M$. Then

$$
\begin{aligned}
\left|\left(v_{j}-v, h-h_{K}\right)\right|=\left|\sum_{k=1}^{K} \alpha_{k}\left(v_{j}-v, e_{k}\right)\right| & \leq \max _{1 \leq k \leq K} 2^{k}\left|\alpha_{k}\right| \sum_{k=1}^{K} \frac{\left|\left(v_{j}-v, e_{k}\right)\right|}{2^{k}} \\
& <\varepsilon / 2
\end{aligned}
$$

for $j>M$. Also, $\left|\left(v_{j}-v, h_{K}\right)\right| \leq 2 C\left\|h_{K}\right\|<\varepsilon / 2$. Therefore,

$$
\left|\left(v_{j}-v, h\right)\right|<\varepsilon, \quad \forall j>M,
$$

that is, $v_{j} \rightarrow v$ weakly in $N$.

\section{REFERENCES}

[1] S. Alama and Y.Y. Li, Existence of solutions for semilinear elliptic equations with indefinite linear part. J. Differential Equations 96 (1992) 89-115.

[2] S. Alama and Y.Y. Li, On "multibump" bound states for certain semilinear elliptic equations. Indiana J. Math. 41 (1992) 983-1026.

[3] T. Bartsch and Y. Ding, On a nonlinear Schrödinger equation with periodic potential. Math. Ann. 313 (1999) 15-37.

[4] V. Benci and G. Cerami, Existence of positive solutions of the equation $-\Delta u+a(x) u=u^{(N+2) /(N-2)}$ in $\mathbf{R}^{N}$. J. Funct. Anal. 88 (1990) 90-117.

[5] B. Buffoni, L. Jeanjean and C.A. Stuart, Existence of nontrivial solutions to a strongly indefinite semilinear equation. Proc. Amer. Math. Soc. 119 (1993) 179-186.

[6] J. Chabrowski and A. Szulkin, On a semilinear Schrödinger equation with critical Sobolev exponent. Preprint of Stockholm University.

[7] J. Chabrowski and J. Yang, On Schrödinger equation with periodic potential and critical Sobolev exponent. Topol. Meth. Nonl. Anal. 12 (1998) 245-261.

[8] V. Coti-Zelati and P.H. Rabinowitz, Homoclinic type solutions for a semilinear elliptic PDE on $\mathbf{R}^{N}$. Comm. Pure Appl. Math. 45 (1992) 1217-1269.

[9] N. Dunford and J.T. Schwartz, Linear Operators. Part I. Interscience (1967).

[10] L. Jeanjean, Solutions in spectral gaps for a nonlinear equation of Schrödinger type. J. Differential Equations 112 (1994) 53-80. 
[11] L. Jeanjean, On the existence of bounded Palais-Smale sequences and application to a Landesman-Lazer type problem set on $\mathbf{R}^{N}$. Proc. Roy. Soc. Edinburgh Sect. A 129 (1999) 787-809.

[12] W. Kryszewski and A. Szulkin, Generalized linking theorem with an application to a semilinear Schrödinger equation. Adv. Differential Equations 3 (1998) 441-472.

[13] P. Kuchment, Floquet Theory for Partial Differential Equations. Birkhäuser, Basel (1993).

[14] Y.Y. Li, On $-\Delta u=K(x) u^{5}$ in $\mathbf{R}^{3}$. Comm. Pure Appl. Math. 46 (1993) 303-340.

[15] Y.Y. Li, Prescribing scalar curvature on $S^{n}$ and related problems. Part I. J. Differential Equations 120 (1995) 319-410.

[16] P.L. Lions, The concentration-compactness principle in the calculus of variations. The locally compact case. Part II. Ann. Inst. H. Poincaré Anal. Non Linéaire 1 (1984) 223-283.

[17] M. Reed and B. Simon, Methods of modern mathematical physics, Vol. IV. Academic Press (1978).

[18] M. Schechter, Critical point theory with weak-to-weak linking. Comm. Pure Appl. Math. 51 (1998) 1247-1254.

[19] M. Schechter, Ratationally invariant periodic solutions of semilinear wave equations. Preprint of the Department of Mathematics, University of California (1998).

[20] M. Schechter, Linking Methods in Critical Point Theory. Birkhäuser, Boston (1999).

[21] M. Struwe, The existence of surfaces of constant mean curvature with free boundaries. Acta Math. 160 (1988) 19-64.

[22] C.A. Stuart, Bifurcation into Spectral Gaps. Bull. Belg. Math. Soc. Suppl. (1995).

[23] A. Szulkin and W. Zou, Homoclinic orbits for asymptotically linear Hamiltonian systems. J. Funct. Anal. 187 (2001) $25-41$.

[24] C. Troestler and M. Willem, Nontrivial solution of a semilinear Schrödinger equation. Comm. Partial Differential Equations 21 (1996) 1431-1449.

[25] M. Willem and W. Zou, On a semilinear Dirichlet problem and a nonlinear Schrödinger equation with periodic potential. Indiana Univ. Math. J. 52 (2003) 109-132.

[26] M. Willem, Minimax Theorems. Birkhäuser, Boston (1996).

[27] W. Zou, Solitary Waves of the Generalized Kadomtsev-Petviashvili Equations. Appl. Math. Lett. 15 (2002) 35-39.

[28] W. Zou, Variant Fountain Theorems and their Applications. Manuscripta Math. 104 (2001) 343-358.

[29] M. Schechter, Some recent results in critical point theory. Pan Amer. Math. J. 12 (2002) 1-19.

[30] M. Schechter and W. Zou, Homoclinic Orbits for Schrödinger Systems. Michigan Math. J. 51 (2003) 59-71.

[31] M. Schechter and W. Zou, Superlinear Problem. Pacific J. Math. (accepted).

[32] W. Zou and S. Li, New Linking Theorem and Elliptic Systems with Nonlinear Boundary Condition. Nonl. Anal. TMA 52 (2003) 1797-1820. 